\title{
A SIMPLE ILLUSTRATION OF A WEAK SPECTRAL CASCADE*
}

\author{
DAVID J. MURAKI ${ }^{\dagger}$
}

\begin{abstract}
The textbook first encounter with nonlinearity in a partial differential equation (PDE) is the first-order wave equation: $u_{t}+u u_{x}=0$. Often referred to as the inviscid Burgers equation, this equation is familiar to many in the theoretical contexts of characteristics, wavebreaking, or shock propagation. Another canonical behavior contained within this simplest of PDEs is the spectral cascade. Surprisingly, buried in a little-known 1964 article by G.W. Platzman is an elegant example of an exact Fourier series solution associated with a purely sinusoidal initial condition. This Fourier representation, valid prior to wavebreaking, is generalized to arbitrary continuous initial conditions on both the periodic and infinite domains. For the specific example of Platzman's original problem, the Fourier coefficients decay exponentially with increasing wavenumber, and the decay rate flattens to zero precisely at the time of wavebreaking. It is demonstrated that two simplified descriptions, a downscale truncation and a linearization from initial conditions, also produce an exponential spectral cascade uniformly to large wavenumbers. This weak cascade is responsible for the initial generation of Fourier harmonics in the viscous Burgers equation.
\end{abstract}

Key words. spectral cascade, nonlinear wave, inviscid Burgers equation

AMS subject classifications. 35L60, 76M45

DOI. $10.1137 / 040619090$

1. Introduction. One of the first nonlinear partial differential equations (PDEs) typically encountered in the applied mathematical canon is the wave equation

$$
u_{t}+u u_{x}=0
$$

which, though elementary, provides a rich introduction to nonlinearity. As a first-order PDE, it provides an example with exact representations for the quasi-linear characteristics. Convergence of these characteristics leads to wavebreaking, multivaluedness, and the development of shock structures. Subsequent propagation of discontinuities is governed by Rankine-Hugoniot conditions obtained from conservation law properties of weak solutions. Beyond this, there is a vast literature associated with this equation whose early references include the simple wave of advection in one-dimensional fluid flow [7], the inviscid limit of the Burgers equation [2], and the kinematic wavespeed equation [21].

Without the advantages of linearity, the usual applications of Fourier methods do not generate modal solutions to (1.1). Rather the opposite occurs, as the forward time evolution from a sinusoidal initial condition, via the wave steepening process, immediately generates a solution with nonzero Fourier amplitudes at all scales. This is an example of a spectral cascade, whereby the nonlinear interaction of Fourier modes leads to an increase in the Fourier amplitudes at shorter spatial scales (higher wavenumbers). While this imagery of the downscale cascade is quite intuitive, as the textbook Fourier methods do not apply to nonlinear PDEs, the absence of illustrative examples is one barrier to elementary-level analysis of this process. It is relatively

\footnotetext{
*Received by the editors November 17, 2004; accepted for publication (in revised form) May 4, 2007; published electronically August 24, 2007. This work was supported by NSERC RGPIN-238928 and NSF CMG-0327658.

http://www.siam.org/journals/siap/67-5/61909.html

${ }^{\dagger}$ Department of Mathematics, Simon Fraser University, Burnaby, BC, V5A 1S6 Canada (muraki@ math.sfu.ca).
} 
unknown, however, that a Fourier series solution, whose coefficients are expressed as Bessel functions, can be elegantly derived for the evolution of (1.1) in the special case of a sinusoidal initial condition. This surprising result, by Platzman in 1964, appeared in Tellus, a journal for dynamic meteorology and oceanography [15].

In this article, we generalize this result to obtain an integral representation of the Fourier coefficients for arbitrary periodic initial conditions, which is valid up to the time of first wavebreaking. This gives an exact formula for each Fourier amplitude as a function of wavenumber and time, which requires only a spatial quadrature over the initial condition. For the wave equation (1.1), the Fourier spectrum is characterized by an exponential decay with wavenumber [18]. The increase in the decay rate with time is a convenient measure of the developing cascade. The weak cascade process is further investigated from the perspectives of spectral dynamics and linearized PDE dynamics about small amplitude initial conditions. For the specific case of sinusoidal initial conditions, both these perspectives on the cascade dynamics also produce shorttime approximations where the exponential decay of the Fourier spectrum is uniform to large wavenumbers. The inviscid cascade is shown to be consistent with initial growth of the exponential spectra observed for the viscous Burgers equation. Finally, the Fourier solution of the wave equation is extended to the infinite line, where it is applied to the downscale cascade from a Gaussian initial condition.

The primary intent here is the presentation of explicit PDE solutions which illustrate the downscale cascade. First, we examine an exact integral formula for obtaining the Fourier coefficients of solutions to the nonlinear wave initial value problem (1.1). Additionally, the special Platzman solution, whose Fourier coefficients are expressible using Bessel functions, provides a benchmark against which we can compare various approximate descriptions of the cascade process. As it happens, the concepts required to relate this particular story nearly read as an introductory syllabus of applied mathematics: characteristics, Fourier representations, special functions, perturbation series, contour integration, and integral asymptotics. So, in keeping with the illustrative nature of this problem, these calculations have been presented in a manner to emphasize its more expository aspects.

2. From characteristics to Fourier series. Consider the general initial value problem of the nonlinear wave equation

$$
u_{t}+u u_{x}=0, \quad u(x, 0)=f(x),
$$

periodic on a domain $-\pi \leq x \leq \pi$. The characteristics are curves in $x-t$ space which are defined by the ordinary differential equation (ODE)

$$
\frac{d x}{d t}=u, \quad x(0)=x_{0},
$$

where $x_{0}$ labels the originating initial point at $(x, t)=\left(x_{0}, 0\right)$. Along this characteristic, the PDE (2.1) is now seen to be the perfect derivative

$$
\frac{d u}{d t}=0, \quad u(0)=f\left(x_{0}\right),
$$

which shows that $u$ maintains the constant value established at its initial point $\left(x_{0}, 0\right)$. Solutions to the ODEs (2.2) and (2.3) produce the wave solution

$$
u=f\left(x_{0}\right), \quad x=u t+x_{0},
$$




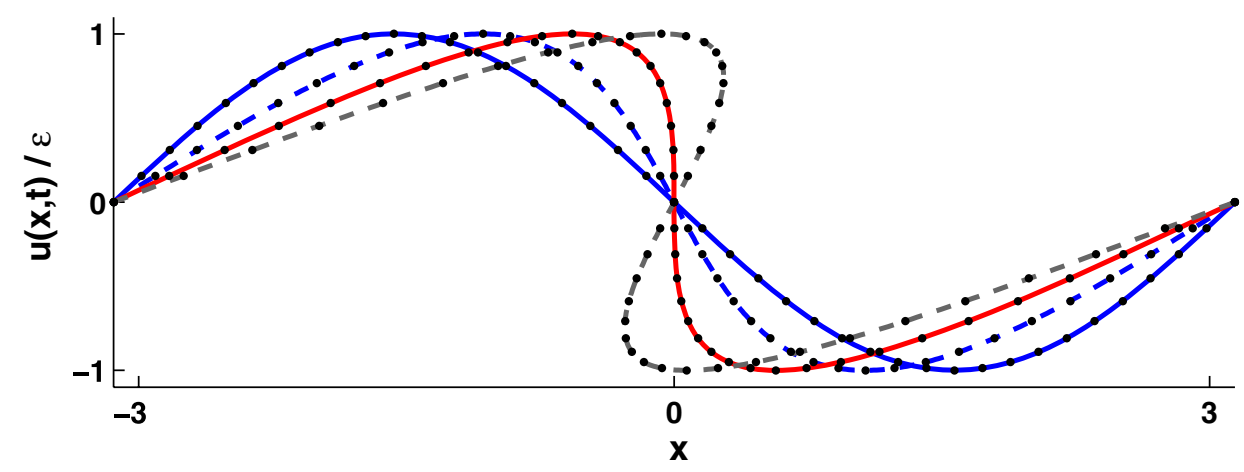

FIG. 2.1. Wavebreaking evolution of $u(x, t) / \epsilon$ beginning from a sinusoidal initial condition $(2.6)$. Shown are scaled times $\epsilon t=0,1 / 2,1,3 / 2$ with the initial and critical wavebreaking profiles in solid and an overturning profile in gray dashed. Obtained from the parametric solution (2.4), the dots track values of $u=f\left(x_{0}\right)$ corresponding to characteristics labeled by $x_{0}$ at intervals of $\pi / 20$.

expressed as a parametrization on $x_{0}$. Eliminating the parameter immediately produces the well-known implicit general solution for $u(x, t)$ :

$$
u=f(x-u t) .
$$

It is a consequence of the nonlinearity in (1.1) that (nontrivial) solutions beginning from smooth initial conditions will eventually develop a finite-time derivative singularity. Figure 2.1 shows the solution $u(x, t)$ beginning from the sinusoidal initial condition

$$
f(x)=-\epsilon \sin x
$$

at times $\epsilon t=0,1 / 2,1,3 / 2$, where the critical wavebreaking event occurs at $\epsilon t_{c}=1$. Although the $\epsilon$ can be removed by rescaling, it is retained for future convenience in the short-time analyses in later sections.

At first glance, construction of a Fourier series solution directly from the PDE (1.1) seems unlikely since nonlinearity precludes the usual application of Fourier transforms. It is a truly remarkable consequence from Platzman's original analysis that the Fourier series representation of $u(x, t)$,

$$
\begin{aligned}
u(x, t) & =\frac{a_{0}}{2}+\sum_{n=1}^{\infty}\left[a_{n}(t) \cos n x+b_{n}(t) \sin n x\right], \\
a_{n}(t) & =\frac{1}{\pi} \int_{-\pi}^{+\pi} u(x, t) \cos n x d x, \\
b_{n}(t) & =\frac{1}{\pi} \int_{-\pi}^{+\pi} u(x, t) \sin n x d x,
\end{aligned}
$$

has coefficients $a_{n}(t)$ and $b_{n}(t)$, which can be manipulated into integrals completely determined by the given initial profile $f(x)$. For the sine coefficient $b_{n}(t)$, this reformulation begins from an integration by parts of (2.9), followed by a replacement of $u_{x}$ using the parametrized form of the characteristic $x=u t+x_{0}(2.4)$, 


$$
\begin{aligned}
b_{n}(t) & =\frac{1}{\pi n} \int_{-\pi}^{+\pi} u_{x}(x, t) \cos n x d x \\
& =\frac{1}{\pi n t} \int_{-\pi}^{+\pi}\left(1-\frac{d x_{0}}{d x}\right) \cos n x d x .
\end{aligned}
$$

Noting that only the $d x_{0} / d x$-term contributes to the full-period integration, changing the variable of integration to $x_{0}$ gives

$$
b_{n}(t)=-\frac{1}{\pi n t} \int_{-\pi}^{+\pi} \cos \left[n x_{0}+n t f\left(x_{0}\right)\right] d x_{0}
$$

and achieves a final integral which involves only the initial condition (2.1). Analogous operations obtain the cosine coefficients for $n \geq 0$ :

$$
a_{n}(t)= \begin{cases}\frac{1}{\pi} \int_{-\pi}^{+\pi} f\left(x_{0}\right) d x_{0} & \text { for } n=0, \\ \frac{1}{\pi n t} \int_{-\pi}^{+\pi} \sin \left[n x_{0}+n t f\left(x_{0}\right)\right] d x_{0} & \text { for } n>0\end{cases}
$$

where the exceptional $n=0$ case is simply the conservation of the mean by the PDE (1.1). It is important to note that the use of integration by parts assumes that the solution remains continuous and hence is not valid after wavebreaking.

A further step can be taken by substituting the Fourier coefficients (2.12) and (2.11) back into the series (2.7). First, the Fourier sine and cosine sums collapse into a single sum

$$
\begin{aligned}
u(x, t) & =\frac{a_{0}}{2}+\sum_{n=1}^{\infty} \frac{1}{\pi n t} \int_{-\pi}^{+\pi} \sin n\left[x-x_{0}-t f\left(x_{0}\right)\right] d x_{0} \\
& =\frac{a_{0}}{2}+\frac{1}{t} \int_{-\pi}^{+\pi}\left[\left(\frac{x-x_{0}-t f\left(x_{0}\right)}{2 \pi} \bmod 1\right)-\frac{1}{2}\right] d x_{0}
\end{aligned}
$$

then an interchange of sum and integral yields what seems to be a quadrature solution for (2.1). Prior to crossing of characteristics, however, $u(x, t)$ cannot depend globally on the initial condition, but is determined exactly by one value of the initial condition. The resolution of this apparent nonlocality is the presence of the modulus in (2.13), which produces a discontinuous integrand. The discontinuity occurs precisely at the unique value of $x_{0}$ parametrizing the characteristic (2.4) that determines $u(x, t)$. Shifting the integration domain to the periodic interval $x_{0}-2 \pi \leq y \leq x_{0}$ allows the removal of the modulus

$$
\begin{aligned}
u(x, t) & =\frac{a_{0}}{2}+\frac{1}{t} \int_{x_{0}-2 \pi}^{x_{0}}\left[\frac{x-y-t f(y)}{2 \pi}-\frac{1}{2}\right] d y \\
& =\left[\frac{a_{0}}{2}-\frac{1}{2 \pi} \int_{x_{0}-2 \pi}^{x_{0}} f(y) d y\right]+\int_{x_{0}-2 \pi}^{x_{0}}\left[\frac{x-x_{0}}{2 \pi t}-\frac{y-\left(x_{0}-\pi\right)}{2 \pi t}\right] d y \\
& =\frac{x-x_{0}}{t}=f\left(x_{0}\right)
\end{aligned}
$$

and, after some grouping of terms, reduces the integral to the local value $f\left(x_{0}\right)$. Similarly, for the case when several characteristics are involved, the integral then 


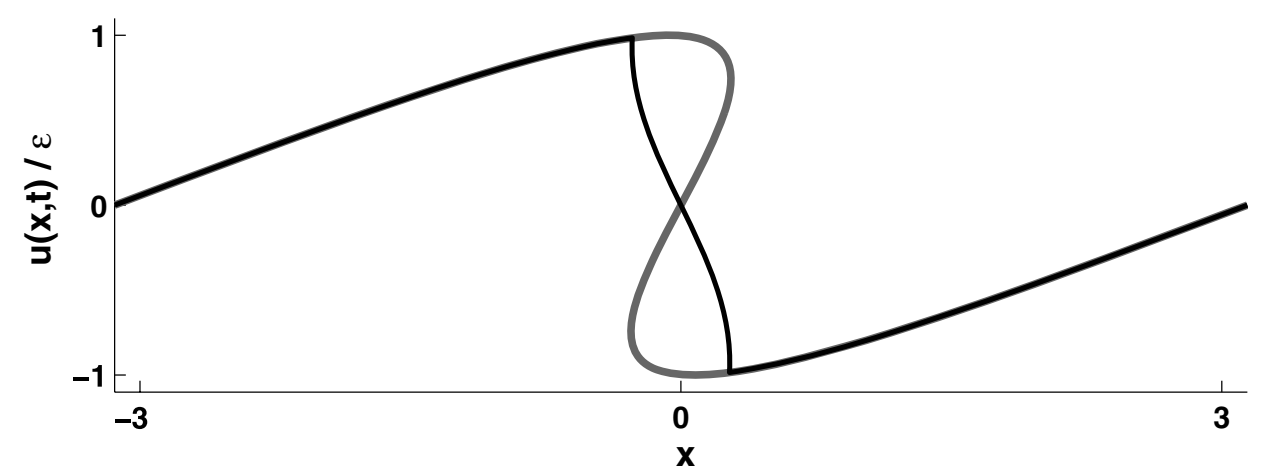

FIG. 2.2. The characteristic solution (2.4) from Figure 2.1 (thick, light curve) beyond the wavebreaking time $(\epsilon t=3 / 2)$ compared with the Fourier series representation (3.2), which is single-valued and continuous (thin, dark curve). The two solutions differ only in regions where the characteristic solution is multivalued.

becomes a weighted sum over all such characteristic values $\pm f\left(x_{0}\right)$, where the sign matches that of $d x / d x_{0}$. For instance, when the characteristic solution becomes triplevalued $\left(u_{b}<u_{m}<u_{t}\right)$, the series adopts the value $u_{b}-u_{m}+u_{t}$. This averaging effect within the Fourier series is illustrated by the thin dark curve in Figure 2.2, in comparison to the multivalued characteristic solution $(\epsilon t=3 / 2)$ as replicated from Figure 2.1. Thus, although the Fourier series defined by (2.11) and (2.12) no longer satisfies the original PDE (1.1) after wavebreaking, the series retains a meaning related to the multivaluedness of the characteristic solution (2.4), but not one connected with any of the usual entropy solutions [8].

3. Platzman's solution and its downscale cascade. The specific example considered by Platzman [15] was based upon the sinusoidal initial condition (2.6), whose forward evolution is shown as Figure 2.1. It is this solution for which Platzman essentially realized that the Fourier coefficient (2.11),

$$
b_{n}(t)=-\frac{1}{\pi n t} \int_{-\pi}^{+\pi} \cos \left(n x_{0}-n t \epsilon \sin x_{0}\right) d x_{0}=-2 \frac{J_{n}(\epsilon n t)}{n t},
$$

resulted in a standard integral representation of the Bessel function of order $n$ [1]. This produced a solution to the nonlinear wave equation (1.1) having an exact expression for its Fourier sine series

$$
u(x, t)=-2 \sum_{n=1}^{\infty} \frac{J_{n}(\epsilon n t)}{n t} \sin n x,
$$

where, in the $t \rightarrow 0^{+}$limit, only the $n=1$ term is nonzero and the initial condition (2.6) is satisfied. In classical analysis, summations whose terms involve Bessel functions of increasing indices and arguments are known as Kapteyn series [19]. As a historical aside, Platzman also recognized that the identical series also appears in the analysis of the Keplerian orbital problem.

It is clear from the coefficients (3.1) that all modes become activated for $t>0$. This is an illustration of a downscale spectral cascade whereby the nonlinear evolution from a single initial Fourier mode leads to the immediate appearance of all smaller 


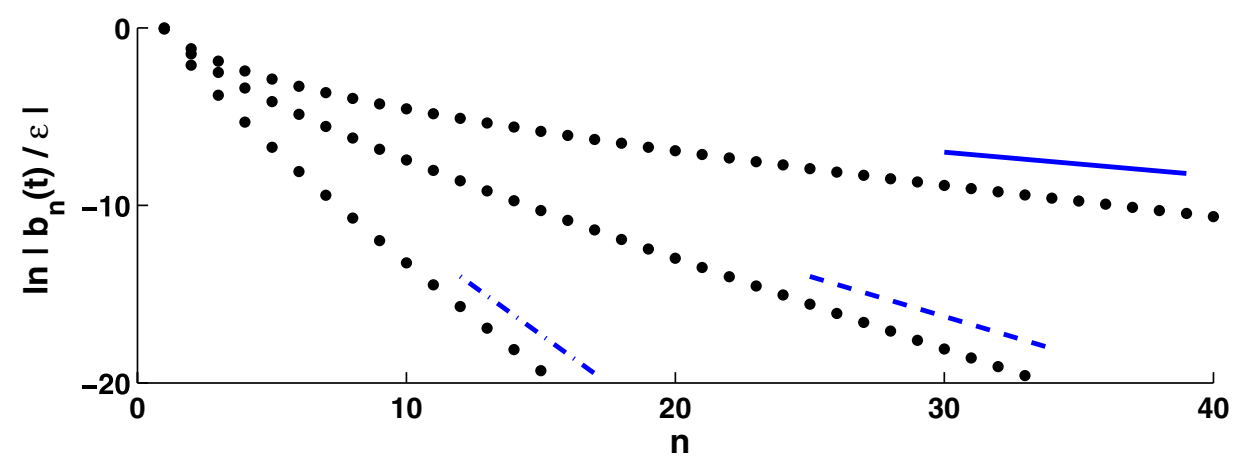

FIG. 3.1. Semilog plot of spectral amplitudes $b_{n}(t) / \epsilon$ for Platzman's Fourier series solution (3.2) showing the growth of the $n>1$ modes at scaled times $\epsilon t=1 / 4,1 / 2,3 / 4$. The exponential spectrum is indicated by linear asymptotes (3.5), which are becoming flatter with time (dash-dot, dash, solid), and thus illustrates a downscale spectral cascade. The decrease in the fundamental $n=1$ mode is not discernable on this semilog axis.

scales. A Bessel recurrence identity [1] gives an alternate expression for (3.1),

$$
b_{n}(t)=-\epsilon \frac{J_{n+1}(\epsilon n t)+J_{n-1}(\epsilon n t)}{n},
$$

from which it follows that the $n \geq 2$ amplitudes $\left|b_{n}(t)\right|$ are strictly increasing up to the time of wavebreaking, since $J_{n}^{\prime}(z)>0$ in the interval $0<z<n$ [19]. The exception is the fundamental amplitude $\left|b_{1}(t)\right|$, the source of the cascade, which decreases steadily and is roughly $88 \%$ of its original amplitude at the time of critical wavebreaking. Figure 3.1 shows a semilog plot of the Fourier amplitudes against wavenumber for the times $\epsilon t=1 / 4,1 / 2,3 / 4$.

Also shown in Figure 3.1 are lines indicating the large- $n$ asymptotic slopes of the semilog spectral amplitudes. These are evident from the Debye expansions for the Bessel functions of large index and argument [1],

$$
\left|b_{n}\right| \sim \sqrt{\frac{2}{\pi t^{2} \tanh \alpha}} n^{-3 / 2} e^{n(-\alpha+\tanh \alpha)} \text { as } n \rightarrow \infty,
$$

where $\cosh \alpha=1 / \epsilon t$. This wavenumber-dependence of the Fourier coefficient reflects the (real) analytic nature of the solution $u(x, t)$ [3]. The spectral slope, $-\alpha+\tanh \alpha$, represents the exponential decay rate with wavenumber and can be explicitly written in terms of $\epsilon t$ :

$$
\frac{\ln \left|b_{n}\right|}{n} \sim \ln \left(\frac{\epsilon t}{2}\right)+\sqrt{1-\epsilon^{2} t^{2}}-\ln \left(\frac{1+\sqrt{1-\epsilon^{2} t^{2}}}{2}\right) \quad \text { as } n \rightarrow \infty .
$$

This expression is equivalent to that deduced by Sulem, Sulem, and Frisch [18] from the pole singularities of the analytic continuation of $u(x, t)$ to the complex $x$-plane. ${ }^{1}$ The early cascade has a spectral slope whose growth is logarithmic in time and corresponds to a geometric decay of the Fourier amplitudes by the factor $\epsilon t$ (as illustrated

\footnotetext{
${ }^{1}$ Also in [18] is the identification of a narrow $n^{-4 / 3}$ spectral regime which occurs just prior to the critical wavebreaking time. This corresponds to a special case of the Bessel asymptotics [1].
} 
later in (5.3)). However, as the wavebreaking $\epsilon t=1$ is approached, the spectral slope flattens to zero. After this time, the decay becomes algebraic following the development of the derivative singularities such as those shown in Figure 2.2.

The connection of (3.1) to a known integral identity appears to be unique to the Platzman initial condition. More generally, however, the form of (2.12) and (2.11) is such that when the integrands are analytic, extraction of the exponential cascade can be approached by deforming the path of integration into the complex $x$-plane. For entire $f(x)$, the method of steepest descent applies, as the integration path can be deformed such that the large $n$ contribution is localized to a saddle point. This approach can also be used to obtain (3.4). The saddle-point method is illustrated by the example for the infinite line formulation in section 7 .

4. Spectral dynamics and the short-time cascade. A conventional approach for analyzing the cascade is by direct substitution of the series (2.7) into the PDE (1.1). For the special case of a Fourier sine series, the terms involved in the $\sin n x-$ mode are

$$
\begin{aligned}
\cdots+b_{n}^{\prime} \sin n x & +\cdots \\
& +\sum_{1}^{n-1} k b_{k} b_{n-k} \cos k x \sin (n-k) x \\
& +\sum_{\substack{1 \\
\infty}} k b_{k} b_{n+k} \cos k x \sin (n+k) x \\
& +\sum_{1}^{\infty}(n+k) b_{n+k} b_{k} \cos (n+k) x \sin k x \quad+\cdots=0
\end{aligned}
$$

After applying a trigonometric product identity and reorganizing the terms, a description of the spectral dynamics is obtained as coupled ODEs:

$$
b_{n}^{\prime}=-\frac{n}{4} \sum_{1}^{n-1} b_{k} b_{n-k}+\frac{n}{2} \sum_{1}^{n-1} b_{k} b_{n+k}+\frac{n}{2} \sum_{n}^{\infty} b_{k} b_{n+k}
$$

for the amplitudes $b_{n}(t)$ over wavenumbers $n$. The first of the three sums corresponds to downscale transfer involving longer waves with wavenumbers from below, $k<n$ and $(n-k)<n$. The second corresponds to mixing transfer involving straddling wavenumbers, $k<n<n+k$, while the third corresponds to upscale transfer involving only shorter waves, $n \leq k<n+k$. These last two summations can be combined into a single sum. It is quite unclear as to how the Bessel amplitudes (3.1) could possibly have been directly obtained beginning only from the spectral ODEs (4.2) and the initial conditions $\left\{b_{n}(0)\right\}=\{-\epsilon, 0,0, \ldots\}$.

Analytical progress is possible, however, in the limit of small $\epsilon$. At $O(1)$ times, the assumption of small amplitude initial condition leads to a wavenumber scaling of the Fourier amplitudes $b_{n}(t)=O\left(\epsilon^{n}\right)$ and allows a natural truncation of the spectral dynamics (4.2) to involve only the downscale transfer summation

$$
\tilde{b}_{n}^{\prime}=\left\{\begin{array}{cc}
0 & \text { for } n=1, \\
-\frac{n}{4} \sum_{1}^{n-1} \tilde{b}_{k} \tilde{b}_{n-k} & \text { for } n \geq 2 .
\end{array}\right.
$$


This will be referred to as the downscale cascade truncation. The exact solution to the above truncation must therefore be the small $\epsilon$ limit of Platzman's solution (3.1),

$$
\tilde{b}_{n}(t)=-\epsilon \frac{n^{n-1}}{n !}\left(\frac{\epsilon t}{2}\right)^{n-1}
$$

which derives from the first nonzero term of the Taylor expansion for the Bessel function [1]. Verification of this, by direct substitution of (4.4) into (4.3), yields a combinatorial identity of uncommon origin - one such instance is found in graph theory as an elementary counting of trees [10]. A direct approach for arriving at expression (4.4) is via a generating function

$$
B(z, t)=\sum_{n=1}^{\infty} \tilde{b}_{n}(t) \frac{e^{i n z}}{2 i} .
$$

By virtue of the downscale spectral dynamics (4.3), $B(z, t)$ also satisfies the same nonlinear wave equation (1.1),

$$
B_{t}+B B_{z}=0, \quad B(z, 0)=\epsilon \frac{e^{i z}}{2 i},
$$

but now with a complex-valued initial condition that is exactly the restriction of the original sinusoid to the positive wavenumber modes. Solution by characteristics leads to the implicit relation

$$
i B t e^{i B t}=\frac{\epsilon t}{2} e^{i z},
$$

whose inversion can be expressed in terms of Lambert's transcendental equation (see also [20]), and otherwise designated by the $W$-function $[6]$,

$$
B(z, t)=-\frac{i}{t} W\left(\frac{\epsilon t}{2} e^{i z}\right) .
$$

However, explicit recovery of the formula for the coefficients (4.4) follows more directly from (4.7) with the application of the Lagrange inversion theorem. Using the Stirling approximation for the factorial in (4.4) gives the large wavenumber behavior

$$
\tilde{b}_{n} \sim-\sqrt{\frac{2}{\pi t^{2}}} n^{-3 / 2} e^{n}\left(\frac{\epsilon t}{2}\right)^{n} \quad \text { for } n \rightarrow \infty
$$

and implies the downscale spectral slope

$$
\frac{\ln \left|\tilde{b}_{n}\right|}{n} \sim \ln \left(\frac{\epsilon t}{2}\right)+1 \quad \text { as } n \rightarrow \infty \text { for } \epsilon t \ll 1 .
$$

The difference here from the full cascade (3.5) is that the slope from the downscale cascade truncation (4.10) is less steep, as the exclusion of any upscale transfers results in a more rapid generation of a smaller-scale spectrum. Hence, at short times $(\epsilon t \ll 1)$, the spectral slope (3.5) for Platzman's example is well described by the downscale cascade approximation (4.3). 
5. A linearized description of the weak cascade. One conclusion from the previous section is that even the truncation of the spectral dynamics to the downscale transfer requires the solution of a fully nonlinear problem. As such, the results relied upon considerable good karma in there being an exact solution (4.4) to a system of nonlinear equations (4.3). In this section, a linear approach is investigated for constructing an approximate solution to the PDE (2.1) that involves the full spectrum of wavenumbers.

Consider a weakly nonlinear analysis which seeks the form of a perturbation expansion

$$
u(x, t) \sim f(x)+u_{2}(x, t)+u_{3}(x, t)+\cdots,
$$

where the first term is a small amplitude initial condition $f(x)=O(\epsilon) \gg u_{2}(x, t) \gg$ $u_{3}(x, t) \ldots$ for $\epsilon \ll 1$. The simplest such expansion assumes that the corrections $u_{n}(x, t)=O\left(\epsilon^{n}\right)$. Substituting (5.1) into the PDE and collecting on powers of $\epsilon$ gives the sequence of equations

$$
\frac{\partial u_{n}}{\partial t}=-\sum_{1}^{n-1} u_{n-k} \frac{\partial u_{k}}{\partial x}, \quad u_{n}(x, 0)=0
$$

which can be solved iteratively for $n \geq 2$ by direct integration for $t>0$. For the sinusoidal initial conditions, the first two corrections are

$$
\begin{aligned}
& u_{2}(x, t)=-\epsilon\left(\frac{\epsilon t}{2}\right) \quad \sin 2 x, \\
& u_{3}(x, t)=-\epsilon\left(\frac{\epsilon t}{2}\right)^{2} \quad\left\{\frac{3}{2} \sin 3 x-\sin x\right\} ;
\end{aligned}
$$

subsequent terms $u_{n}(\underset{\tilde{b}}{x}, t)$ contain only $O\left(\epsilon^{n}\right)$ expressions, which include not only the short-time harmonic $\tilde{b}_{n}(t) \sin n x$ from the downscale transfer (4.4), but also smaller harmonics due to contributions from the straddling and upscale transfers (4.2). Finite application of this method thus produces an $O\left(\epsilon^{n}\right)$ series expansion limited to the first $n$ harmonics. Such a finite expansion is not a uniform approximation over wavenumbers, since for the sinusoidal initial condition the extent to which the spectral cascade is realized is limited by the number of terms in the expansion (5.1).

To develop an approach which involves all harmonics, consider the solution as a disturbance from a small amplitude initial condition

$$
u(x, t)=f(x)+\tilde{u}(x, t),
$$

so that $\tilde{u}(x, t) \ll f(x)=O(\epsilon)$. This results in the exact disturbance equation

$$
\tilde{u}_{t}=-f f_{x}-(f \tilde{u})_{x}-\tilde{u} \tilde{u}_{x}, \quad \tilde{u}(x, 0)=0,
$$

where the right-hand side terms are nominally $O\left(\epsilon^{2}\right), O\left(\epsilon^{3}\right)$, and $O\left(\epsilon^{4}\right)$. If (5.5) is approximated by keeping only the $f f_{x}$-term, then the disturbance $\tilde{u}(x, t)$ is $O\left(\epsilon^{2}\right)$ correct and would be identical to $u_{2}(x, t)$ as determined by (5.2). Alternatively, an additional order in $\tilde{u}(x, t)$ is achieved if only the last and nonlinear disturbance term is neglected. This truncation can be interpreted as a first Newton iterate, since the $\tilde{u}$-correction is obtained by a linearized solve against a residual error (in the form of the $f f_{x}$-term). Thus we consider the linearized problem

$$
U_{t}+(f U)_{x}=-f f_{x}, \quad U(x, 0)=0,
$$


so that $u(x, t) \sim f(x)+U(x, t)$ constitutes an $O\left(\epsilon^{3}\right)$-correct asymptotic representation. Multiplying the equation though by $f(x)$ and defining $v(x, t)=f(x) U(x, t)$ gives

$$
v_{t}+f v_{x}=-\frac{1}{2} f\left(f^{2}\right)_{x}, \quad v(x, 0)=0,
$$

which is a first-order but nonconstant coefficient and inhomogeneous PDE. Unlike the original PDE (1.1), the characteristics for the linearization (5.6) do not depend on the solution, but only on the initial condition, via

$$
\frac{d x}{d t}=f(x), \quad x(0)=x_{0}
$$

where again $x_{0}$ labels the originating initial point at $(x, t)=\left(x_{0}, 0\right)$. Along this characteristic, the PDE (5.8) now becomes the perfect derivative

$$
\frac{d v}{d t}=-\frac{1}{2} \frac{d x}{d t}\left(f^{2}\right)_{x}=-\frac{1}{2} \frac{d\left(f^{2}\right)}{d t}, \quad v\left(x_{0}, 0\right)=0,
$$

which relies upon the $t$-independence of $f^{2}$. Direct integration from a zero initial condition gives the solutions

$$
\begin{aligned}
v(x, t) & =-\frac{1}{2}\left(f^{2}(x)-f^{2}\left(x_{0}\right)\right), \\
U(x, t) & =-\frac{1}{2}\left(\frac{1-f^{2}\left(x_{0}\right)}{f^{2}(x)}\right) f(x),
\end{aligned}
$$

where the label $x_{0}=x_{0}(x, t)$ is obtained by inverting the solution of the characteristic ODE (5.8). Specifically for Platzman's initial condition, it is shown next that this correction term is no longer spectrally limited to a few harmonics but embodies a cascade across all wavenumbers.

For the case of $f(x)=-\epsilon \sin x$, the characteristic ODE (5.8) is a nonlinear but separable equation; hence

$$
\ln \left(\frac{\tan x / 2}{\tan x_{0} / 2}\right)=\int_{x_{0}}^{x} \frac{d x}{\sin x}=-\epsilon \int_{0}^{t} d t=-\epsilon t,
$$

from which the trigonometric relation $\tan \left(x_{0} / 2\right)=e^{\epsilon t} \tan (x / 2)$ follows. Using this and a half-angle identity gives

$$
\sin x_{0}=\frac{2 \tan \left(x_{0} / 2\right)}{1+\tan ^{2}\left(x_{0} / 2\right)}=\frac{2 e^{\epsilon t} \tan (x / 2)}{1+e^{2 \epsilon t} \tan ^{2}(x / 2)}=\frac{\operatorname{sech} \epsilon t}{1-\tanh \epsilon t \cos x} \sin x,
$$

which, in the Platzman case, effects the inversion of the characteristic label $x_{0}$ into the original $x, t$-coordinates. Thus, the linearized solution (5.10) leads to the asymptotic approximation

$$
u(x, t) \sim-\epsilon \sin x+\frac{\epsilon}{2}\left(1-\frac{\operatorname{sech}^{2} \epsilon t}{(1-\tanh \epsilon t \cos x)^{2}}\right) \sin x+O\left(\epsilon^{4}\right),
$$

where the second term is actually $O\left(\epsilon^{2}\right)$ with the vanishing of the bracketed factor when $\epsilon=0$. 
Obtaining the spectral cascade requires finding the Fourier series representation of (5.13). The obvious problematic term is the second term in the correction whose Fourier-sine coefficient is the imaginary part of

$$
-\frac{\epsilon}{2 \pi} \operatorname{sech}^{2} \epsilon t \int_{-\pi}^{+\pi} \frac{e^{i n x} \sin x}{(1-\tanh \epsilon t \cos x)^{2}} d x .
$$

This expression can be evaluated via complex contour integration around a rectangle whose corners are $\{-\pi,+\pi,+\pi+i Y,-\pi+i Y\}$. Contributions from the sides parallel to the imaginary axis cancel by the periodicity of the integrand, and the contribution from the side with $\operatorname{Im}(z)=Y$ tends to zero as $Y \rightarrow+\infty$ by the decay of the integrand. The closed contour contains only a double pole at $z_{p}$, where

$$
\begin{aligned}
\cos z_{p} & =\cosh i z_{p}=\frac{\tanh (\epsilon t 2)+\operatorname{coth}(\epsilon t 2)}{2}=\operatorname{coth} \epsilon t \\
& \Rightarrow \quad e^{i z_{p}}=\tanh (\epsilon t 2),
\end{aligned}
$$

and thus is located along the positive imaginary axis for $\epsilon t>0$. The end result of this residue calculation is the series representation for (5.13):

$$
u(x, t) \sim-\frac{\epsilon}{2}\left(\sin x+\operatorname{sech}^{2}(\epsilon t 2) \sum_{1}^{\infty} n \tanh ^{n-1}(\epsilon t 2) \sin n x\right),
$$

which reveals that again the spectrum has exponential decay, whose spectral slope is

$$
\text { slope } \sim \ln \left|\tanh \frac{\epsilon t}{2}\right| \quad \text { as } n \rightarrow \infty \text { for } \epsilon t \ll 1 .
$$

Thus the logarithmic part of the $\epsilon t \ll 1$ spectral slope is obtained. It is emphasized that this is really just a scaling result on the amplitudes, indicating only that harmonics decay as powers of $\epsilon t$. This limited result is not too surprising since the downscale cascade within the short-time and linear approximation is still a fully nonlinear process (4.3). Nonetheless, for the sinusoidal initial condition, the linearization (5.6) does produce, after just one perturbative calculation, an explicit short-time correction (5.13) that is asymptotically valid only to $O\left(\epsilon^{3}\right)$ yet captures the exponential decay of the spectrum uniformly to large wavenumbers.

More generally, the linearization generates Fourier harmonics through the action of the nonconstant coefficient (5.7), as the Fourier harmonics are no longer the linear modes. In essence, this allows a coupling between Fourier coefficients, but one where the strength of the interaction is determined by the nonconstant coefficient and hence is fixed in time by the initialized state. The above example shows that even when the coupling is established by an initial condition consisting only of a single initial sinusoid, the cascade described by (5.6) captures the downscale cascade with an exponential decay in wavenumber. A second example of a linearized cascade based on a spectrally richer initial Gaussian is computed for the infinite line case of section 7 .

6. The weak cascade of the Burgers equation. The exponential spectrum appears in the wave equation (1.1) as a consequence of pole singularities associated with the analytic continuation of the inversion (2.5) over complex $x$ [18]. An exponential spectrum is also common to solutions of the Burgers equation [16, 18], and it includes the effect of viscous, linear dissipation into the nonlinear wave equation [2]:

$$
u_{t}+u u_{x}=\sigma u_{x x}, \quad u(x, 0)=f(x) .
$$



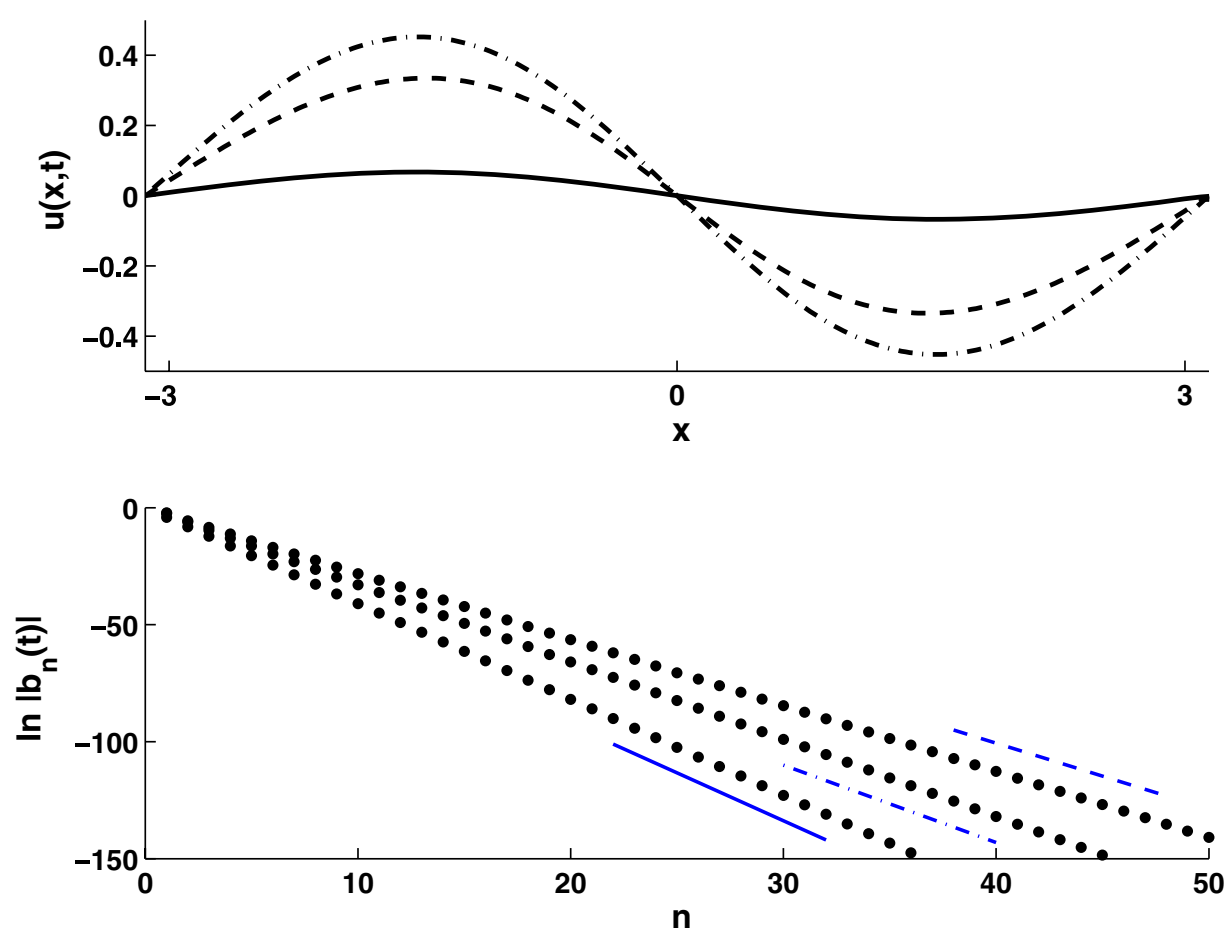

FIG. 6.1. Top panel shows a Burgers solution beginning from a sinusoidal initial condition for $\epsilon=0.5, \sigma=1.0$ at times $t=0.1,0.4,2.0$ (dash-dot, dashed, solid). Bottom panel shows the corresponding semilog Fourier spectra with the asymptotic slopes indicated. The spectral slope initially increases until roughly $t=0.4$, and then decreases linearly in time.

A familiar spectral result $[2,18,4]$ is the exponential spectrum for the steady-state tanh-solution. It is also well known that the Burgers dynamics is equivalent to the linear diffusion equation via the Hopf-Cole transformation [21],

$$
u=-2 \sigma \frac{\psi_{x}}{\psi}, \quad \psi_{t}=\sigma \psi_{x x} .
$$

For Hopf-Cole functions $\psi(x, t)$ which are meromorphic over complex $x$, the evaluation of Fourier coefficients

$$
b_{n}(t)=-\frac{2 \sigma}{\pi} \operatorname{Imag} \int_{-\pi}^{+\pi} \frac{\psi_{x}(x, t)}{\psi(x, t)} e^{i n x} d x
$$

by a contour integration of the type used to obtain (5.14) involves only simple poles. The residue of the pole with smallest imaginary part determines the spectral slope of the exponential spectrum [18].

The upper panel of Figure 6.1 shows the decay of the Burgers solution beginning from an initial sinusoid (2.6) of small amplitude $(\epsilon=0.5, \sigma=1.0)$ as computed by a fully spectral code. Clearly apparent in the lower panel of Figure 6.1 are the linear asymptotes in the corresponding semilog plots of Fourier amplitudes. Inspection of the chronology of the spectra (dash-dot, dashed, solid) reveals that the spectral slopes initially increase in time, and subsequently decrease through the action of viscous dissipation. Figure 6.2 (solid) shows the best-fit spectral slopes, as a function of 


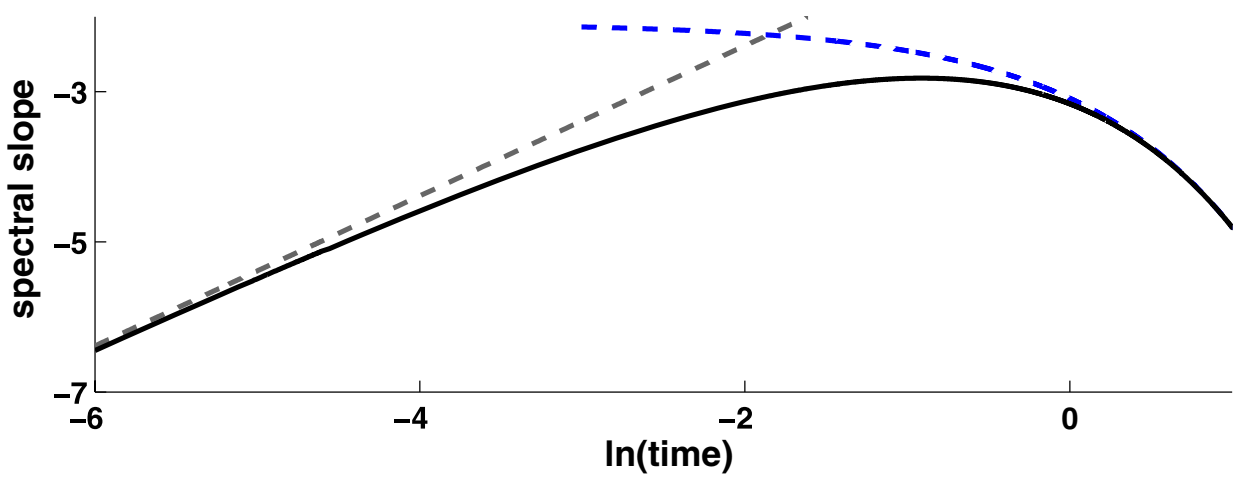

FIG. 6.2. The semilog spectral slope as a function of log-time for the evolution of Figure 6.1 as obtained by the best-fit line over modes $n=80-100$ (solid). At short times, the Platzman spectral slope (4.10) illustrates that the growth of the initial cascade scales similarly as the inviscid dynamics (gray dashed). At long times, the slope follows the asymptote as obtained from the Hopf-Cole solution (6.8) and shows the erosion of the spectrum by the viscous decay (dashed).

$\log$-time, for the evolution of Figure 6.1. In terms of the spectral slope, the Burgers cascade exhibits the same growth as the inviscid cascade (4.10) at very early times (gray dashed). At later times, when the amplitudes are decaying, the spectral slope approaches a linear-in-time asymptote (dashed). It is not apparent how to obtain the early-time spectral growth directly from the Hopf-Cole solution, but the long-time behavior is easily extracted.

The initial Hopf-Cole function is given by

$$
\psi(x, 0)=\exp \left(-\frac{\epsilon}{2 \sigma} \cos x\right)=I_{0}\left(\frac{\epsilon}{2 \sigma}\right)+2 \sum_{1}^{\infty} I_{n}\left(-\frac{\epsilon}{2 \sigma}\right) \cos n x,
$$

where the series representation [1] involves a modified Bessel identity which is closely related to that used in the Platzman cascade (3.1). The time-dependent evolution thus has the exact Fourier solution

$$
\psi(x, t)=I_{0}\left(\frac{\epsilon}{2 \sigma}\right)+2 \sum_{1}^{\infty} I_{n}\left(-\frac{\epsilon}{2 \sigma}\right) e^{-\sigma n^{2} t} \cos n x .
$$

After sufficiently long times (regardless of the values of $\epsilon$ and $\sigma$ ), the Hopf-Cole dynamics is dominated by the slowest decaying $n=1$ mode,

$$
\psi(x, t) \sim I_{0}\left(\frac{\epsilon}{2 \sigma}\right)-2 I_{1}\left(\frac{\epsilon}{2 \sigma}\right) e^{-\sigma t} \cos x .
$$

The corresponding Burgers solution then has the form

$$
\begin{aligned}
u(x, t) & \sim-4 \sigma \frac{I_{1}(\epsilon / 2 \sigma) e^{-\sigma t} \sin x}{I_{0}(\epsilon / 2 \sigma)-2 I_{1}(\epsilon / 2 \sigma) e^{-\sigma t} \cos x} \\
& =-4 \sigma \sum_{1}^{\infty} e^{n \rho} \sin n x,
\end{aligned}
$$

where the spectral slope, $\rho$, calculated using the same contour used to obtain (5.14), is given by

$$
\rho=-\cosh ^{-1}\left(\frac{I_{0}(\epsilon / 2 \sigma)}{2 I_{1}(\epsilon / 2 \sigma)} e^{\sigma t}\right) \sim-\sigma t+\ln \left(\frac{I_{1}(\epsilon / 2 \sigma)}{I_{0}(\epsilon / 2 \sigma)}\right) \quad \text { for } t \gg 1 .
$$


This is the long-time asymptote (dashed) shown in Figure 6.2 and is the generic spectral behavior once the Burgers evolution is dominated by a largest scale mode.

The special case of the sinusoidal initial condition illustrates that the initial downscale cascade evolves on the inviscid timescale, $\epsilon t$, despite the fact that the advective term is a weakly nonlinear effect. The cascade then transitions to dissipation on the viscous timescale, $\sigma t$, as the solution comes to be dominated by the slowest decaying mode $u(x, t) \sim e^{-\sigma t} \sin x$. The modal dissipation is then driven by weak nonlinearity, as harmonics decay as $e^{-n \sigma t}$, in contrast to the $e^{-n^{2} \sigma t}$ decay of linear diffusion.

7. Fourier solution on the infinite line. The derivation of the periodic Fourier coefficients (2.11), (2.12) is easily modified to obtain an analogous integral for the Fourier transform solution on the infinite line. Defining the Fourier transform representation of continuous solutions to (1.1) by

$$
u(x, t)=\int_{-\infty}^{+\infty} c(k, t) e^{-i k x} d k
$$

the coefficients $c(k, t)$ can also be shown to derive from the initial profile $u(x, 0)=$ $f(x)$. Beginning from the Fourier integral, an integration by parts is performed,

$$
c(k, t)=\frac{1}{2 \pi} \int_{-\infty}^{+\infty} u(x, t) e^{i k x} d x=\frac{i}{2 \pi k} \int_{-\infty}^{+\infty} u_{x}(x, t) e^{i k x} d x
$$

which again assumes continuity as well as sufficiently fast decay of the solution at $x \rightarrow \pm \infty$. In a slight departure from the periodic case, the next step introduces the parametric solution $u=f\left(x_{0}\right)$,

$$
\begin{aligned}
c(k, t) & =\frac{i}{2 \pi k} \int_{-\infty}^{+\infty} f^{\prime}\left(x_{0}\right) \frac{d x_{0}}{d x} e^{i k x} d x \\
& =\frac{i}{2 \pi k} \int_{-\infty}^{+\infty} f^{\prime}\left(x_{0}\right) \exp \left[i k\left(x_{0}+t f\left(x_{0}\right)\right)\right] d x_{0},
\end{aligned}
$$

where decay of the integrand is ensured through the initial profile. (Note that an analogous formula can also be derived for the periodic case.)

For example, the solution from an initial Gaussian profile $f(x)=e^{-x^{2} / 2}$ remains single-valued up until the breaking time of $t_{c}=\sqrt{e}$ (Figure 7.1). In the limit of large wavenumber $k$, the Fourier integral (7.3) can be approximated by the method of steepest descent. The complex plane for the phase function $\phi(z)=i\left(z+t e^{-z^{2} / 2}\right)$ is shown as Figure 7.2. The saddle points of the phase are determined by the stationary points $\phi^{\prime}\left(z_{s}\right)=0$, which for the Gaussian profile can be expressed as the condition

$$
\left(-z_{s}^{2}\right) e^{\left(-z_{s}^{2}\right)}=-\frac{1}{t^{2}}
$$

Thus the saddle points are complex-valued solutions to Lambert's transcendental equation $z_{s}^{2}=-W\left(-1 / t^{2}\right)$. Figure 7.2 shows the four saddle points closest to the real $z$-axis at time $t / t_{c}=3 / 4$. The integration along the real axis (7.3) can be deformed into a scalloped contour (solid curve in Figure 7.2) in the upper half-plane, so that the dominant contribution will be localized to the saddle point with the maximum $\operatorname{Re}\left(\phi\left(z_{s}\right)\right)$. The quadratic Taylor expansion of the phase function at a saddle point simplifies to

$$
\phi(z) \sim i\left(z_{s}+\frac{1}{z_{s}}\right)+\frac{i}{2}\left(z_{s}-\frac{1}{z_{s}}\right)\left(z-z_{s}\right)^{2}
$$




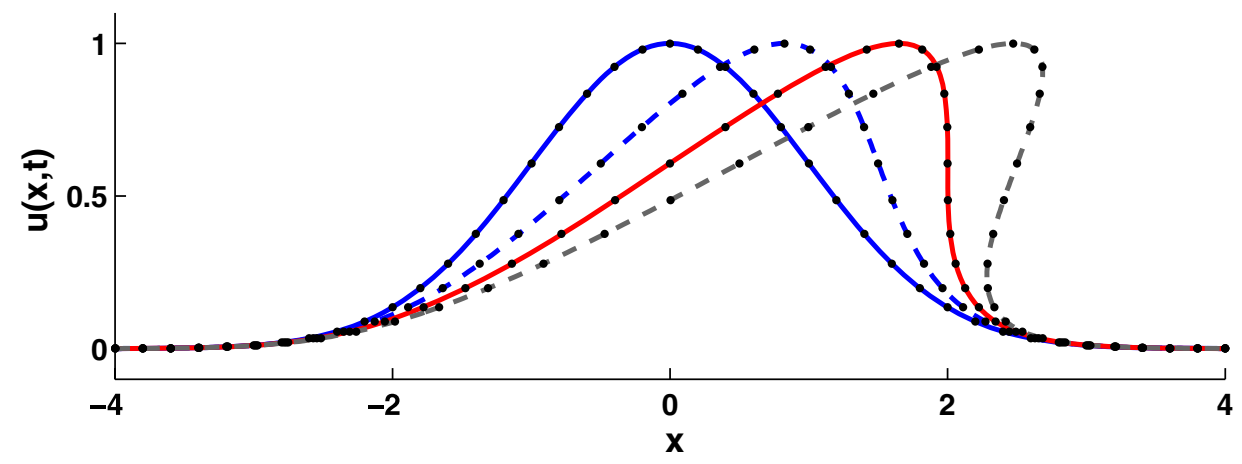

FIG. 7.1. Wavebreaking evolution of $u(x, t) / \epsilon$ beginning from a Gaussian initial condition. Shown are scaled times $t / t_{c}=0,1 / 2,1,3 / 2$ with the initial and critical wavebreaking profiles in solid and an overturning profile in gray dashed. Obtained from the parametric solution (2.4), the dots track values of $u=f\left(x_{0}\right)$ corresponding to characteristics labeled by $x_{0}$ at intervals of $1 / 5$.

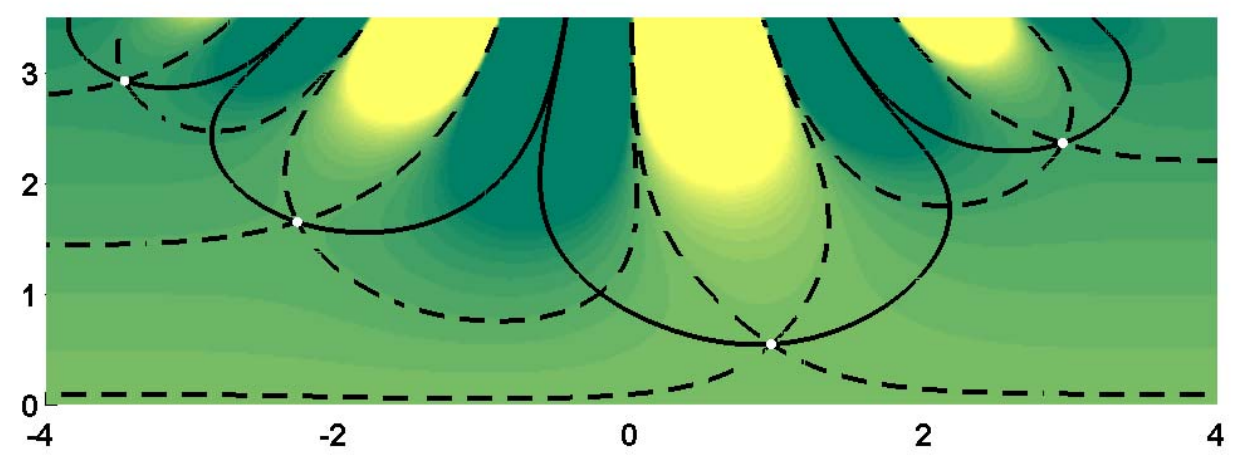

FIG. 7.2. Complex plane for the phase function $\phi(z ; t)$ for $t / t_{c}=3 / 4$. The grayscale indicates $\operatorname{Re}(\phi(z ; t))$, where darker regions correspond to exponential smallness of the integrand. The contours shown are associated with the four saddle points closest to the real axis (closest $z_{s} \approx 0.95+0.55 i$ ). Solid contours are paths of steepest descent to regions of exponentially small integrand. Dashed contours are level curves of the magnitude.

and gives the steepest descent contribution

$$
\sqrt{\frac{1}{2 \pi i\left(z_{s}-1 / z_{s}\right) t^{2}}} k^{-3 / 2} e^{i\left(z_{s}+1 / z_{s}\right) k},
$$

where additional time-dependence lies in the location of the saddle point (7.4). Using only the dominant saddle point, this gives an expression for the spectral slope:

$$
\frac{\ln |c(k, t)|}{k} \sim-\operatorname{Im}\left(z_{s}+\frac{1}{z_{s}}\right) \text { as } k \rightarrow \infty,
$$

which is verified by the lines in Figure 7.3. As expected, the breaking time $t_{c}=$ $\sqrt{e}$ coincides with the first real root of the saddle-point condition (7.4), where the spectral decay changes from exponential to algebraic. Thus, at finite times $0<t<t_{c}$, the spectrum decays exponentially despite its beginning from more rapid quadratic Gaussian decay (light curve). 


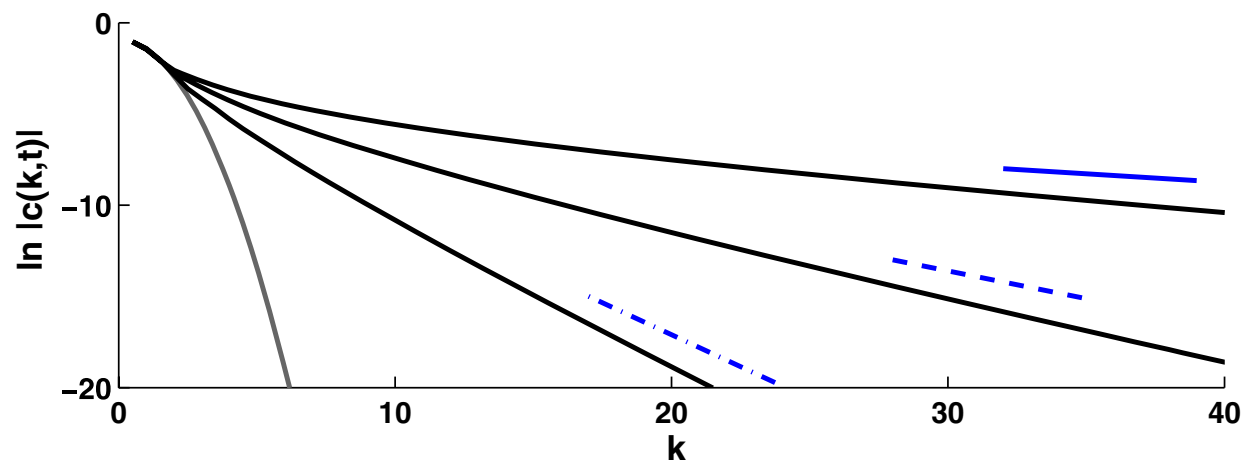

FIG. 7.3. Semilog plot of spectral amplitudes $c_{n}(t) / \epsilon$ of a pseudospectral computation (de-aliased to 2048 modes on a $4 \pi$-periodic domain) from a Gaussian initial condition (leftmost curve). The downscale spectral cascade is illustrated by the growth of the Fourier amplitudes (other dark solid) over times $t / t_{c}=1 / 4,1 / 2,3 / 4$. The flattening of the exponential spectrum is indicated by the linear asymptotes (dash-dot, dash, solid) as calculated from the steepest descent contribution (7.7) from the saddle point nearest the real axis.

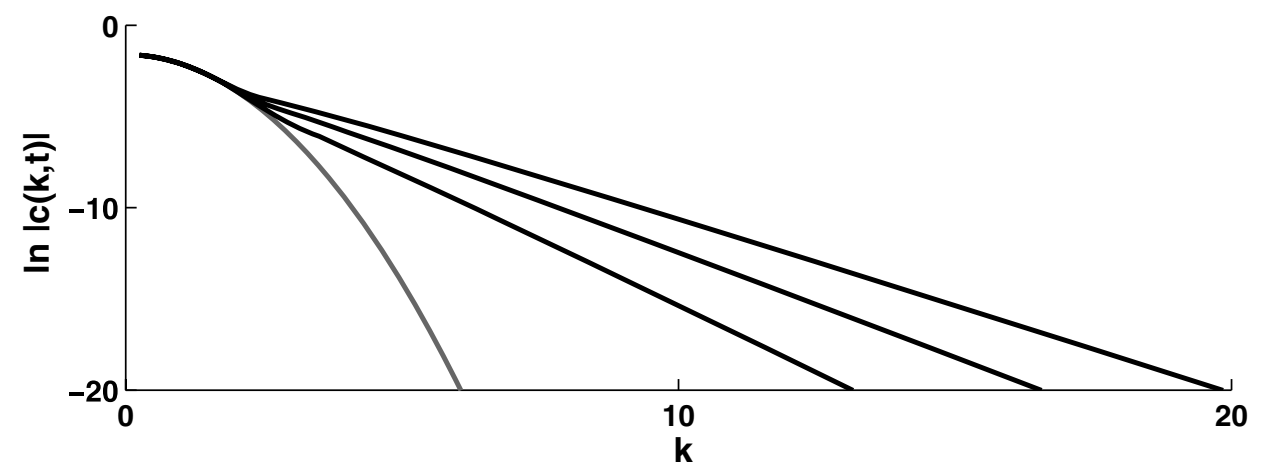

FIG. 7.4. Semilog plot of spectral amplitudes $c_{n}(t) / \epsilon$ for the linearized evolution (5.6) from an initial Gaussian (gray solid) shows that its cascade also develops an exponential spectrum. The numerical parameters are the same as those of the nonlinear computation of Figure 7.3. The flattening of the spectrum is shown for earlier times $t / t_{c}=1 / 12,1 / 6,1 / 4$, based on the breaking time of the nonlinear evolution. The slope of the linearized cascade at $t=t_{c} / 4$ lags just behind the fully nonlinear cascade shown in Figure 7.3.

The development of the linearized spectrum from the Gaussian initial condition is shown in Figure 7.4. Although the communication between the Fourier modes is fixed by the spectral characteristics at the initial time in (5.6), the linear evolution still generates a downscale cascade with an exponential decay with wavenumber.

8. Closing thoughts. The Fourier results presented here are spectral identities which follow from Platzman's observation that $u_{x}$ is simply related to the change of variable $d x_{0} / d x$ via the parametric solution (2.4) for (1.1). This is a rather unusual situation that does not readily apply beyond the characteristic wave equation. Nonetheless, one generalization for which such spectral formulas can be stated is

$$
u_{t}+g(u, x) u_{x}=h^{\prime}(t) u, \quad u(x, 0)=f(x) .
$$


On the infinite line, the Fourier transform is expressible as the integral

$$
c(k, t)=\frac{i}{2 \pi k} e^{h(t)-h(0)} \int_{-\infty}^{+\infty} f^{\prime}\left(x_{0}\right) e^{i k x\left(x_{0}, t\right)} d x_{0},
$$

where $x\left(x_{0}, t\right)$ is determined by the characteristic ODE

$$
\frac{d x}{d t}=g\left(f\left(x_{0}\right) e^{h(t)-h(0)}, x\right), \quad x(0)=x_{0} .
$$

The occurrence of nontrivial examples where the formulas (8.2), (8.3) allow further analysis is a rare event. However, a decaying version of Platzman's example with the additional effect $h^{\prime}=-\alpha$, a constant value, yields

$$
b_{n}(t)=\frac{2 \alpha}{n\left(1-e^{\alpha t}\right)} J_{n}\left(\frac{\epsilon n\left(1-e^{-\alpha t}\right)}{\alpha}\right),
$$

an exercise that reveals a suppression of the wavebreaking when $\alpha>\epsilon$.

As an explicit Fourier analysis of the inviscid Burgers equation, the spectral formulas (2.12), (2.11), (7.3) can be used to investigate cascades from other initial conditions. Although Platzman's example highlighted the downscale cascade, the dynamics of upscale transfers to large scales from smaller scales can also be addressed by initial sums of sinusoids. For real analytic initial conditions, the method of steepest descent generally applies for obtaining the asymptotic exponential spectrum. In contrast, compactly supported (but piecewise continuous) initial conditions yield simple examples whose spectra have algebraic decay.

The Burgers equation, and its inviscid limit, have long been used as a pedagogical introduction to the nonlinearity of fluid motions $[2,5,9]$. This generalization of Platzman's result provides explicit formulas for one-dimensional, deterministic, and continuous realizations of the spectral cascade - hence the qualification to a weak cascade. Large wavenumber asymptotics for the integral formula allow direct calculation of the exponential decay of the Fourier spectrum. The inviscid cascade is observed to characterize the early development of the spectrum in the viscous Burgers equation. The examples shown here are direct spectral illustrations of the nonlinear cascade but are far from those required to understand the multidimensional, statistical nature of fluid turbulence. Nonetheless, they are a clear demonstration of a nonlinear process by which advection can sustain an exponential spectrum in the dissipation range, the scales at which the fields are smooth $[17,12]$. The turbulence question aside, these results provide an elementary contribution to the broader effort to understand the spectral signatures of singularities in nonlinear PDEs $[14,18,16]$.

Although the existence of an exact solution (2.5) would seemingly render (1.1) as fully understood, recent revisitations in the research literature remind that this textbook equation still serves as a source of inspiration for investigations in nonlinearity. Weideman [20] used complex-valued solutions as tests for a method for tracking singularities using numerical analytical continuation. In particular, the dynamics of the logarithmic branch point was computed for the initial condition $u(x, 0)=e^{i x}$. The sinusoidal initial condition has also been used by Majda and Timofeyev [11] to initiate postbreaking ergodic dynamics among the Fourier modes for a Galerkin truncation of the spectral dynamics. The truncated dynamics exhibits a chaos which is shown to have a well-defined statistical equilibrium. Finally, the linearization result is very closely related to some current investigations of Mattingly, Soudian, and VandenEijnden [13], who are constructing linear spectral cascade models with exact solutions. 
These models have spectral dynamics which are limited to linear coupling of nearest neighbors (in wavenumber), of which (5.6) is an inviscid example. Their analyses involve an unexpected generalization of the generating function method that is based on orthogonal eigenfunction expansions.

In these investigations the wave equation (1.1) is utilized as a testbed for furthering our understanding of nonlinearity. It is in a similar spirit that these one-dimensional Fourier results, although limited to continuous solutions, are communicated for their novelty as an exact spectral viewpoint.

Acknowledgments. The author acknowledges Ed Spiegel, who recommended the Platzman article some years ago. Special thanks to colleagues Ralf Wittenberg, J. F. Williams, and Youngsuk Lee for their enthusiastic discussions during the course of this work, and for their careful readings of the manuscript. Finally, the author is very grateful to Jim Colliander [18], Elef Gkioulekas [12], and Mike Siegel [14] for alerting me to related works.

\section{REFERENCES}

[1] M. Abramowitz and I. Stegun, Handbook of Mathematical Formulas and Tables, National Bureau of Standards, Washington, DC, 1964.

[2] J. M. Burgers, A Mathematical Model Illustrating the Theory of Turbulence, Adv. Appl. Mech. 1, Academic Press, New York, 1948.

[3] G. F. Carrier, M. Krook, and C. E. Pearson, Functions of a Complex Variable, McGrawHill, New York, 1966.

[4] A. J. ChORIN AND O. H. HALd, Viscosity-dependent inertial spectra of the Burgers and Korteweg-deVries-Burgers equation, Proc. Natl. Acad. Sci. USA, 102 (2005), pp. 3921-3923.

[5] J. D. COLE, On a quasi-linear parabolic equation occurring in aerodynamics, Quart. Appl. Math., 9 (1951), pp. 225-236.

[6] R. M. Corliss, G. H. Gonnet, D. E. G. Hare, D. J. Jeffere, and D. E. Knuth, On the Lambert W Function, Adv. Comput. Math., 5 (1996), pp. 339-359.

[7] R. Courant and K. O. Friedrichs, Supersonic Flow and Shock Waves, Springer-Verlag, New York, 1948.

[8] L. C. Evans, Partial Differential Equations, American Mathematical Society, Providence, RI, 1998.

[9] E. Hopf, The partial differential equation $u_{t}+u u_{x}=\nu u_{x x}$, Comm. Pure Appl. Math., 3 (1950), pp. 201-230.

[10] D. E. Knuth And B. Pittel, A recurrence related to trees, Proc. Amer. Math. Soc., 105 (1989), pp. 335-349.

[11] A. J. Majda and I. Timofeyev, Remarkable statistical behavior for truncated Burgers-Hopf dynamics, Proc. Natl. Acad. Sci. USA, 97 (2000), pp. 12413-12417.

[12] O. P. Manley, The dissipation range spectrum, Phys. Fluids A, 4 (1992), pp. 1320-1321.

[13] J. C. Mattingly, T. M. Suidan, and E. Vanden-Eijnden, J. Statist. Phys., to appear.

[14] D. W. MoOre, The spontaneous appearance of a singularity in the shape of an evolving vortex sheet, Proc. Roy. Soc. London Ser. A, 365 (1979), pp. 105-119.

[15] G. W. Platzman, An exact integral of complete spectral equations for unsteady onedimensional flow, Tellus, 16 (1964), pp. 422-431.

[16] D. Senouf, Dynamics and condensation of complex singularities for Burgers' equation II, SIAM J. Math. Anal., 28 (1997), pp. 1490-1513.

[17] L. M. Smith And W. C. ReYnolds, The dissipation-range spectrum and the velocity-derivative skewness in turbulent flows, Phys. Fluids A, 3 (1991), pp. 992-994.

[18] C. Sulem, P.-L. Sulem, And H. Frisch, Tracing complex singularities with spectral methods, J. Comput. Phys., 50 (1983), pp. 138-161.

[19] G. N. Watson, A Treatise on the Theory of Bessel Functions, Cambridge University Press, Cambridge, UK, 1944.

[20] J. A. C. WeIDEman, Computing the dynamics of complex singularities of nonlinear PDEs, SIAM J. Appl. Dyn. Syst., 2 (2003), pp. 171-186.

[21] G. B. Whitham, Linear and Nonlinear Waves, Wiley, New York, 1974. 